\title{
1q42.12q42.2 Deletion in a Child with Midline Defects and Hypoplasia of the Corpus Callosum
}

\author{
Akella Radha Rama Devi ${ }^{a} \quad$ Aparna Ganapathy ${ }^{c}$ Ashraf U. Mannanc \\ Shefali Sabharanjak ${ }^{c}$ Shaik M. Naushad ${ }^{b}$ \\ aRainbow Children's Hospital and 'bSandor Speciality Diagnostics Pvt Ltd, Hyderabad, and \\ 'Strand Center for Genomics and Personalized Medicine, Strand Life Sciences, Bangalore, India
}

\section{Established Facts}

- The earlier reports on 1q42.12q42.2 deletion highlighted the importance of WDR26 and FBXO28 mutations in the phenotypic presentation.

\section{Novel Insights}

- In the current study, the reported deletion is novel and encompasses genes with a high probability of loss-of-function intolerance score, i.e., $L B R, E N A H, A C B D 3$, ITPKB, CDC42BPA, and TAF5L, and hence intolerant to haploinsufficiency. The deletion of these genes explains the phenotypic presentation.

\section{Keywords \\ GNPAT $\cdot L B R \cdot$ pLI score $\cdot 1 \mathrm{q} 42.12 \mathrm{q} 42.2$ deletion}

\section{Abstract}

Chromosome 1q42.12q42.2 deletions are documented as "disease causing" and show overlapping phenotypes depending on the genes involved in the deletion. In this report, we detected a 5.8-Mb deletion encompassing the chromosome 1 q42.12q42.2 region in a 4-year-old boy with hypoplastic corpus callosum, epilepsy, developmental delay, microcephaly, cataract, cleft palate, and skeletal changes. The deletion was de novo. Genotype-phenotype correlations suggest that the major features of 1q42.12q42.2 microdeletion were attributed to the genes with a high probability of loss-of-function intolerance score in this deletion, namely $L B R, E N A H, A C B D 3, L I N 9, I T P K B, C D C 42 B P A, A R F 1, T A F 5 L$, GALNT2, SPRTN, and EGLN1 along with GNPAT.

(c) 2019 S. Karger AG, Basel

Deletions in the chromosomal 1q41q44 region are significantly associated with central nervous system defects including neural tube defects, agenesis of corpus callosum, 
microcephaly, and hydrocephalus. The first case of 1q43 terminal deletion was in a 3-year-old female child [Mankinen et al., 1976]. The 1q42.3qter deletion was attributed to a specific syndrome, "multiple congenital anomalies/mental retardation," based on the phenotype analysis of 23 affected children [Meinecke and Vögtel, 1987].

Seven cases with de novo deletions of 1q41q42 were reported to have the smallest region of overlap of $1.17 \mathrm{Mb}$ encompassing 5 genes, including DISP1, whose deletion was associated with holoprosencephaly in mice. Although none of these patients had holoprosencephaly, many had midline defects such as cleft palate, diaphragmatic hernia, along with seizures, mental retardation, and developmental delay [Shaffer et al., 2007]. DISP1 haploinsufficiency alone may not be responsible for all the major features of 1q41q42 syndrome, other genes in the smallest region of overlap are likely to contribute towards the phenotype [Rosenfeld et al., 2011].

A de novo $5.45-\mathrm{Mb}$ deletion exclusively in $1 \mathrm{q} 42$ was reported to be associated with midline defects including agenesis of the corpus callosum, epilepsy and developmental delay [Filges et al., 2010]. This deletion has a significant overlap with 1q41q42 syndrome with deletion extending into the 1q41 region. These findings suggested that interactions between genes regulating pathways of embryonic development might be contributing to phenotypic presentation rather than haploinsufficiency of single genes. The deletions have been reported to be de novo with different breakpoints collectively named as " $1 \mathrm{q} 4$ deletion syndrome." Every person with a 1q4 deletion is unique and will have different manifestations. Dysmorphic features, seizures, and cognitive disability or developmental delay are present in all patients at varying degrees. Within the spectrum of 1q4 deletion syndrome, deletions restricted to $1 \mathrm{q} 42$ are rare [He et al., 2017]. The 1q4 deletion syndrome is likely to be a contiguous gene deletion syndrome with several causative genes within the region that influences the phenotype. The current study substantiates the abovementioned statement by highlighting the contribution of genes in 1q42, which are less known.

Heterozygous deletions in $1 \mathrm{q} 42.12 \mathrm{q} 42.2$ are documented to be "disease causing" with clinical manifestations such as developmental delay, cognitive disability, dysmorphic features, failure to thrive, microcephaly, and seizures. The size of the deletions can vary from a very small deletion of $400 \mathrm{~kb}$ to large deletions of more than $20 \mathrm{Mb}$. Here, we report a de novo 1q42.12q42.2 microdeletion in a 4 -year-old boy with microcephaly and midline anomaly.

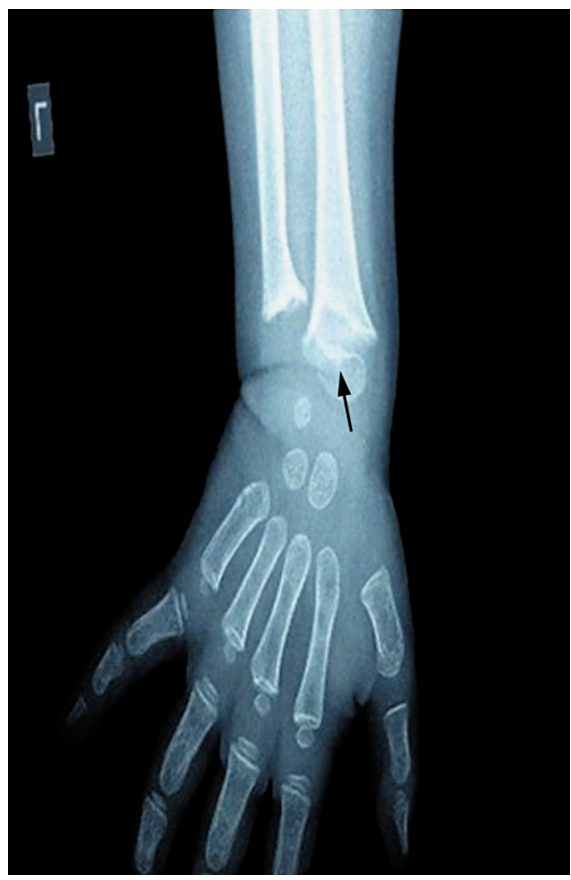

Fig. 1. Anteroposterior view of the proband's wrist showing epiphyseal changes in the long bones. Arrow indicates widened epiphysis.

\section{Case Report}

A 4-year-old firstborn male child of healthy nonconsanguineous parents was evaluated for developmental delay. The child was born by cesarean section at 38 weeks of gestation following an uneventful pregnancy with a birth weight of $2.6 \mathrm{~kg}$. The child was diagnosed to have bilateral cataracts and a cleft palate. Feeding difficulty was present due to the cleft palate in the neonatal period requiring the use of a specialized cleft palate feeding bottle. Visual impairment was observed due to the presence of bilateral cataracts. At the age of 6 months, he underwent cataract surgery and surgical correction of the cleft palate. Subsequently, his parents noticed delayed milestones associated with seizures. Visual maturation did not improve even after cataract surgery. At 12 months, global psychomotor retardation was present with severe sensorineural hearing loss. At 4 years of age, growth was at the $3 \mathrm{rd}$ centile with evident short stature. Seizures were refractory, and he was totally confined to bed. Except for frontal bossing and short stature as well as a widened epiphysis at the wrist, he had no other dysmorphisms. Brain MRI showed hypoplastic corpus callosum. Skeletal examination showed evidence of epiphyseal changes in the long bones (Fig. 1). A provisional diagnosis of Lowe syndrome was suspected because of cataracts, skeletal changes, and intellectual disability. Next-generation sequencing (NGS) was performed, and a 1q4 deletion was identified. Further confirmation was done by real-time quantitative polymerase chain reaction (qPCR) and deletion analysis. Based on the exome results, a peripheral blood count was done which showed hypolobulation in the neutrophils. Parents were not carriers for the deletion. The couple underwent amniocentesis in view of the possibility of germline/gonadal mosaicism in the next pregnancy. Fetal DNA analysis revealed an unaffected fetus. 
Table 1. Comparison of current case and reported cases with deletions in similar regions

\begin{tabular}{llll}
\hline Clinical feature & Current case & $\begin{array}{l}\text { Garza-Flores } \\
\text { et al. (2017) }\end{array}$ & $\begin{array}{l}\text { Filges } \\
\text { et al. (2010) }\end{array}$ \\
\hline Deleted region, bp & $225590996_{-}$ & $222253867-$ & 221885000 \\
Birth weight, kg & 231413298 & 229251861 & 227340000 \\
Corpus callosum agenesis & +6 & 2.8 & 2.89 \\
Cleft palate & + & + & + \\
Intellectual disability & + & + & + \\
Sensorineural hearing loss & + & + & + \\
Delayed milestones & + & + & - \\
Visual impairment & + & + & + \\
Laryngotracheal abnormality & - & + & + \\
Congenital heart disease & - & + & + \\
Refractory seizures & + & + & - \\
Skeletal defects & + & + & + \\
Diaphragmatic hernia & - & + & - \\
De novo & + & - & + \\
\hline
\end{tabular}

\section{Materials and Methods}

\section{Next-Generation Sequencing}

NGS was performed on the patient's genomic DNA using TruSight One panel (Illumina, San Diego, CA, USA) that covers coding exons and flanking intronic sequences of $>4,800$ genes associated with known inherited diseases. Genomic DNA was used for the library preparation for NGS as previously described [Mannan et al., 2016] and sequenced on the NextSeq platform (Illumina) according to the manufacturer's instructions. Variations were identified using the STRAND ${ }^{\circledR}$ NGS software and imported into StrandOmics ${ }^{\mathrm{TM}}$, a proprietary clinical genomics interpretation and reporting platform. Copy number analysis was performed to detect large deletions and duplications, by comparing the normalized coverage and $Z$ scores of the sample against a profile of multiple samples run on the NGS panel using STRAND ${ }^{\circledR}$ NGS, as previously described [Singh et al., 2016]. The variants identified were classified according to the American Society of Medical Genetics and Genomics (ACMG) recommendation for standards for interpretation and reporting of sequence variations [Richards et al., 2015].

\section{Confirmation of the Microdeletion by $q P C R$}

Using Primer3 software [Rozen et al., 2000], primers were designed for exon 2 and exon 13 of the $L B R$ gene and exon 2 and exon 15 of the GNPAT gene which represent genes at the distal and proximal ends of the deleted region. The qPCR was performed in triplicate using SYBR Fast Universal Readymix qPCR kit (KAPA, Woburn, MA, USA) in MxPro30005P apparatus (Stratagene, Santa Clara, CA, USA), as per manufacturer's instructions. The exon deletion was determined using the comparative threshold cycle $\operatorname{method}\left(\Delta \Delta \mathrm{C}_{\mathrm{T}}\right)$.

\section{Prenatal Testing}

Fetal genomic DNA was obtained by chorionic villus sampling during the second pregnancy of the index patient's parents and tested for the presence of the identified deletions of the $L B R$ and GNPAT genes using the qPCR method.

1q42.12q42.2 Deletion Syndrome

\section{Results}

The index patient was heterozygous for $1 \mathrm{q} 42.12 \mathrm{q} 42.2$ (chr1:225590996-?_231413298+?del, GRCh37/hg19). In this $5.8-\mathrm{Mb}$ deleted region, the genes with a high probability of loss-of-function intolerance score were: LBR, ENAH, ACBD3, LIN9, ITPKB, CDC42BPA, ARF1, TAF5L, GALNT2, SPRTN, and EGLN1 (Fig. 2). As the LBR (chr1:225589204_225616627) and GNPAT (chr1: 231376919_231413719) are at the most proximal and distal ends of the deletion, the deletion was further confirmed by performing qPCR assay on exon 2 and exon 13 of $L B R$ and exon 2 and exon 15 of GNPAT. Fetal DNA was assessed using qPCR for the same 2 genes, $L B R$ and GNPAT. The deletion was absent in the fetal sample.

\section{Discussion}

The proband in this study exhibited a heterozygous deletion in 1q42.12q42.2 (chr1:225590996_231413298), which has significant overlap with the deletion reported earlier (chr1:222253867_229251861) in an 18-year-old female [Garza-Flores et al., 2017]. Both had low birth weight ( 2.6 vs. $2.8 \mathrm{~kg}$ ) and anomalies reminiscent of Toriello-Carey syndrome such as agenesis of the corpus callosum and a cleft palate. Both shared other features atypical of Toriello-Carey syndrome such as intellectual disability, sensorineural hearing impairment, delayed milestones, refractory seizures, and visual impairment. In contrast to the case presented by Garza-Flores et al. [2017], the WDR26 (chr1:224572845_224624735) and FBXO28 (chr1:224301789_224349749) genes are not part of the deletion in the current study (Table 1).

Cases with deletions in 1q41q42 are rarely reported, and existing evidence mostly showed its correlation with congenital diaphragmatic hernia [Kantarci et al., 2006] and Fryns syndrome. The hernia is not present in our patient as the deletion did not cover the $1 \mathrm{q} 41$ region. Instead, cleft palate, the most frequently reported midline anomaly, is seen in our patient. Corpus callosum agenesis in our patient corroborates with an earlier study on 1q42 deletion reported by Filges et al. [2010] in a mother and her 2 sons. However, it does not include the DISP1 (chr1: 222988406_223179337) gene that is associated with developmental anomalies [Shaffer et al., 2007]. Therefore, we propose that the regions 1q42.11,1q42.12, and 1q42.13 probably contain candidate genes other than DISP1 for midline developmental anomalies such as cleft palate causing benign Pelger-Huet anomaly (OMIM 169400) 


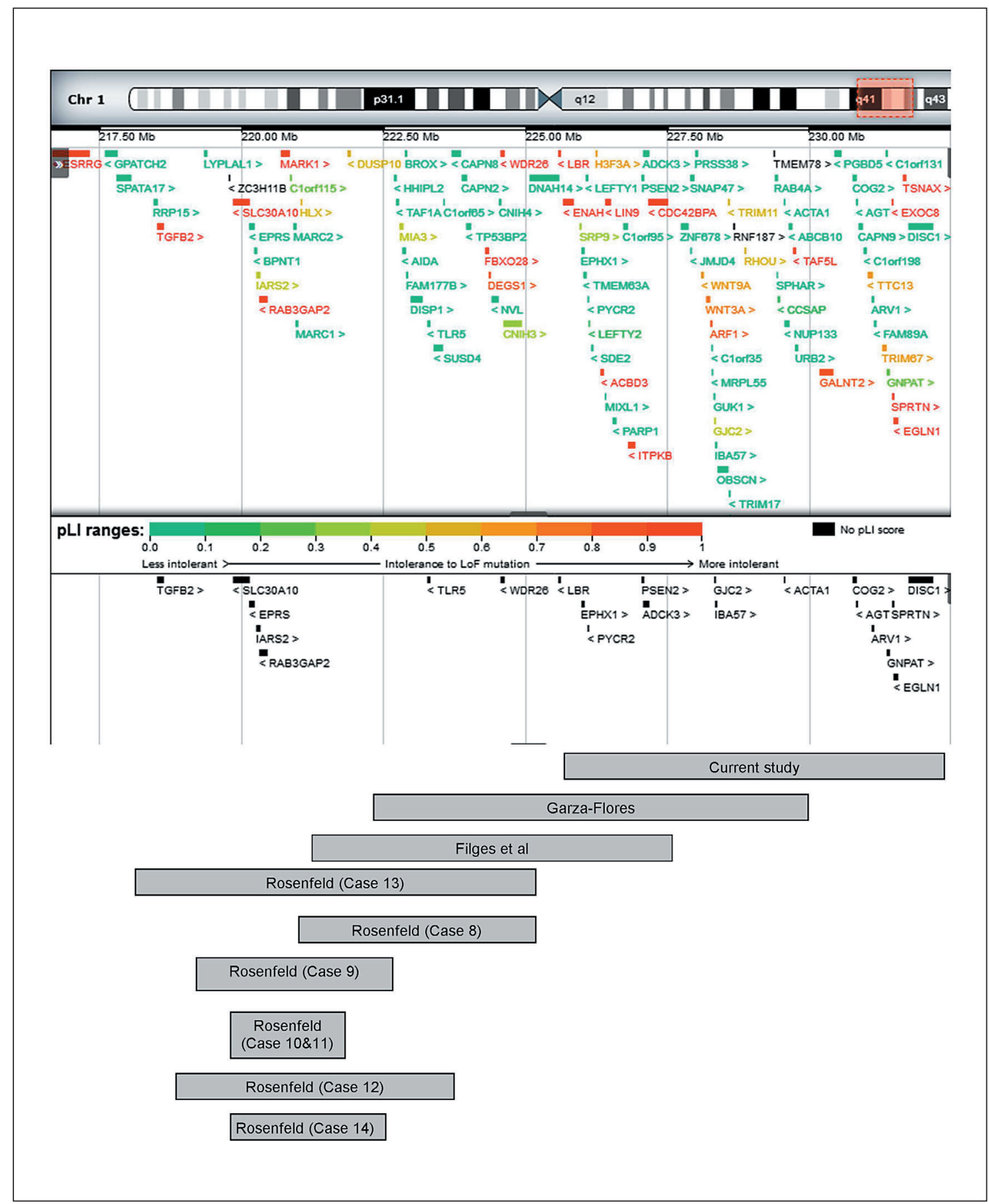

Fig. 2. The comparison of deleted regions across different studies with the current study. The deletion region in our case overlaps with the deletion regions reported by Garza-Flores et al. [2017] and Filges et al. [2010]. No overlap was observed with deletion regions reported by Rosenfeld et al. [2011]. The genes with a high probability of loss-of-function intolerance (pLi) score in this deletion are: LBR, ENAH, ACBD3, LIN9, ITPKB, CDC42BPA, ARF1, TAF5L, GALNT2, SPRTN, and EGLN1. 
and the EPHX1 gene involved in the microsomal epoxide hydrolase defect (OMIM 132810) causing phenytoin treatment toxicity.

Among the genes with a high probability of loss-offunction intolerance score in this deletion, the LBR-encoded lamin B receptor plays an important role in the nuclear envelope [Holmer et al., 1998]. LBR is also associated with 2 different recognized clinical conditions, Pelger-Huet anomaly and Greenberg skeletal dysplasia [Borovik et al., 2013]. Copy number of $L B R$ and the nuclear segmentation index of neutrophils were highly correlated while the gene dosage could affect granulopoiesis [Gravemann et al., 2010]. Our index patient had features of $L B R$ gene deletion manifesting as hypolobularity of the neutrophils (also known as the Pelger-Huet anomaly).

$A C B D 3$ encodes for PMP70, one of the 4 ABC halftransporters of mammalian peroxisomes. RNAi-mediated silencing of $A C B D 3$ gene expression in rat $\mathrm{C} 6$ glial cells was reported to reduce peroxisomal and mitochondrial beta oxidation by one third and also alter the mitochondrial membrane potential [Di Benedetto et al., 2008]. GNPAT gene mutations are associated with rhizomelic chondrodysplasia punctata type2 (RCDP2) manifesting as cataracts, rhizomelia, epiphyseal changes, and intellectual disability. Our patient has all these features except rhizomelia. A phenotype with GNPAT mutation without rhizomelia and cataracts has previously been described [Clayton et al., 1994]. Six additional patients with RCDP2 and RCDP3 due to GNPAT mutations have been identified [Itzkovitz et al., 2012]. GNPAT-associated RCDP2 is inherited in an autosomal recessive mode. It is therefore likely that haploinsufficiency of GNPAT and ACBD3 together might be contributing to the RCDP-like phenotype. We report cataracts as an additional clinical feature in $1 \mathrm{q} 42.12 \mathrm{q} 42.2$ for the first time.

Loss of LIN9 was reported to affect early embryonic development as it regulates mitotic genes such as $P l k 1$, Aurka, and Kif20a [Reichert et al., 2010]. It is likely that the congenital anomalies such as cleft palate and skeletal changes are partially attributed to loss of LIN9. ENAH binds to profilin that modulates actin polymerization. Enah-deficient mice were shown to exhibit defects in neurulation, especially involving the corpus callosum, hippocampus, and pontocerebellar regions [Lanier et al., 1999]. This probably explains the agenesis of corpus callosum in our patient. EGLN1 is a cellular oxygen sensor that catalyzes the post-translational hydroxylation of proline residues in the oxygen-dependent degradation domains of the hypoxia-inducible factor. By regulating the stability of HIF1, it is involved in angiogenesis in retina and cardiac functionality [Takei et al., 2017]. It is likely that deficiency of EGLN1 is contributing to early cataract formation and may also be responsible for loss of visual maturation even after cataract surgery.

We presume that disruption of multiple genes might be contributing to specific phenotypic features discussed in our patient rather than single genes explaining the possibility of a contiguous gene syndrome.

Genetic counseling was provided to the family. Testing results showed that both parents are noncarriers of the mutation, leading to the conclusion that the identified deletion in the patient is de novo. However, due to the parents' anxiety regarding the possibility of gonadal mosaicism, the mother underwent prenatal diagnosis in her next pregnancy. Fetal genomic DNA obtained by chorionic villus sampling was tested, and the pathogenic microdeletion identified in the proband was not detected in the fetus. Pregnancy was continued, and a healthy child was delivered.

\section{Acknowledgments}

We thank the family for their support to evaluate and publish this report and for participating in this study. We also would like to thank Maria Kammerer (Strand Life Sciences) for her editorial contribution to this article.

\section{Statement of Ethics}

The study was approved by the Ethical committee of the Rainbow Children's Hospital, Hyderabad, India. Written informed consent was obtained from the patient's parents.

\section{Disclosure Statement}

The authors declare that they have no conflicts of interest.

\section{Funding Sources}

No specific funding was obtained from any agency or organization for the current study. 


\section{References}

Borovik L, Modaff P, Waterham HR, Krentz AD, Pauli RM: Pelger-Huet anomaly and a mild skeletal phenotype secondary to mutations in LBR. Am J Med Genet A 161A:2066-2073 (2013).

Clayton PT, Eckhardt S, Wilson J, Hall CM, Yousuf $\mathrm{Y}$, et al: Isolated dihydroxyacetonephosphate acyltransferase deficiency presenting with developmental delay. J Inherit Metab Dis 17:533-540 (1994).

Di Benedetto R, Denti MA, Salvati S, Sanchez M, Attorri L, et al: RNAi-mediated silencing of $A B C D 3$ gene expression in rat $\mathrm{C} 6$ glial cells: a model system to study PMP70 function. Neurochem Int 52:1106-1113 (2008).

-Filges I, Röthlisberger B, Boesch N, Weber P, Wenzel F, et al: Interstitial deletion $1 \mathrm{q} 42$ in a patient with agenesis of corpus callosum: phenotype-genotype comparison to the $1 \mathrm{q} 41 \mathrm{q} 42$ microdeletion suggests a contiguous 1q4 syndrome. Am J Med Genet A 152A:987-993 (2010).

Garza-Flores A, Hawley P, Picker J, Tannebring E, Deardorff MA, Lin AE: Response to: Toriello et al., "Update on the Toriello-Carey Syndrome." Further delineation of a young woman with deletion 1q42.12-q42.2. Am J Med Genet A, E-pub ahead of print (2017).

$\checkmark$ Gravemann S, Schnipper N, Meyer H, Vaya A, Nowaczyk, et al: Dosage effect of zero to three functional $L B R$-genes in vivo and in vitro. Nucleus 1:179-189 (2010).

- He J, Xie Y, Kong S, Qiu W, Wang X, et al: Psychomotor retardation with a 1q42.11-q42.12 deletion. Hereditas 154:6 (2017).
Holmer L, Pezhman A, Worman HJ: The human lamin B receptor/sterol reductase multigene family. Genomics 54:469-476 (1998).

Itzkovitz B, Jiralerspong S, Nimmo G, Loscalzo M, Horovitz DD, et al: Functional characterization of novel mutations in GNPAT and AGPS, causing rhizomelic chondrodysplasia punctata (RCDP) types 2 and 3. Hum Mutat 33:189-197 (2012).

- Kantarci S, Casavant D, Prada C, Russell M, Byrne $\mathrm{J}$, et al: Findings from aCGH in patients with congenital diaphragmatic hernia $(\mathrm{CDH})$ : a possible locus for Fryns syndrome. Am J Med Genet A 140:17-23 (2006)

Lanier LM, Gates MA, Witke W, Menzies AS, et al: Mena is required for neurulation and commissure formation. Neuron 22:313-325 (1999).

Mankinen CB, Sears JW, Alvarez VR: Terminal (1)(q43) long-arm deletion of chromosome no. 1 in a three-year-old female. Birth Defects Orig Artic Ser 12:131-136 (1976).

Mannan AU, Singh J, Lakshmikeshava R, Thota N, Singh S, et al: Detection of high frequency of mutations in a breast and/or ovarian cancer cohort: implications of embracing a multigene panel in molecular diagnosis in India. J Hum Genet 61:515-522 (2016).

Meinecke P, Vögtel D: A specific syndrome due to deletion of the distal long arm of chromosome 1. Am J Med Genet 28:371-376 (1987).
Reichert N, Wurster S, Ulrich T, Schmitt K, Hauser S, et al: Lin9, a subunit of the mammalian DREAM complex, is essential for embryonic development, for survival of adult mice, and for tumor suppression. Mol Cell Biol 30: 2896-2908 (2010).

Richards S, Aziz N, Bale S, Bick D, Das S, et al: Standards and guidelines for the interpretation of sequence variants: a joint consensus recommendation of the American College of Medical Genetics and Genomics and the Association for Molecular Pathology. Genet Med 17:405-424 (2015).

Rosenfeld JA, Lacassie Y, El-Khechen D, Escobar LF, Reggin J, et al: New cases and refinement of the critical region in the 1q41q42 microdeletion syndrome. Eur J Med Genet 54:42-49 (2011).

Rozen S, Skaletsky H: Primer3 on the WWW for general users and for biologist programmers. Methods Mol Biol 132:365-386 (2000).

- Shaffer LG, Theisen A, Bejjani BA, Ballif BC, Aylsworth AS, et al: The discovery of microdeletion syndromes in the post-genomic era: review of the methodology and characterization of a new 1q41q42 microdeletion syndrome. Genet Med 9:607-616 (2007).

Singh J, Mishra A, Pandian AJ, Mallipatna AC, Khetan $\mathrm{V}$, et al: Next-generation sequencingbased method shows increased mutation detection sensitivity in an Indian retinoblastoma cohort. Mol Vis 22:1036-1047 (2016).

Takei A, Ekström M, Mammadzada P, Aronsson M, Yu M, et al: Gene transfer of prolyl hydroxylase domain 2 inhibits hypoxia-inducible angiogenesis in a model of choroidal neovascularization. Sci Rep 7:42546 (2017). 